\section{Discussion on: "Dynamic Sliding Mode Control for a Class of Systems with Mismatched Uncertainty"}

\author{
Andrew D. Lewis*
}

$12 / 01 / 2005$

Last updated: $17 / 01 / 2005$

\section{Introduction}

The paper by Yan, Spurgeon, and Edwards uses the standard linear sliding mode procedure - outlined in the books of [Edwards and Spurgeon 1998, Utkin 1992] for examplealong with an observer, to stabilise a class of systems whose linearisation is stabilisable using the standard sliding mode approach, and whose nonlinearities and uncertainties have certain structure. This structure, as well as modifications to the standard observer and controller design, are devised so that the following two criterion are met:

1. standard Lyapunov analysis, using the linear Lyapunov function, should serve to determine the stability of the sliding mode dynamics;

2. standard observer estimates should yield exponential convergence to zero of the error between the estimated and actual state.

Thus the design techniques are in the realm of linear sliding mode control, and the principal contribution as concerns nonlinearities and uncertainties is to characterise the sort of these that are allowable by the linear control design.

In this discussion, I would like to say a few things about sliding mode control in general. Specifically, I would like to compare sliding mode control with standard linear control design techniques, and point out where I think the real advantages of sliding mode control arise I will not touch upon the matter of combining state estimation with sliding mode control; however, it seems plausible to me that all the comments made below carry over, mutatis mutandis, to this situation.

\section{Sliding mode control for linear systems}

Let us consider a MIMO linear system $(\boldsymbol{A}, \boldsymbol{B}, \boldsymbol{C}) \in \mathbb{R}^{n \times n} \times \mathbb{R}^{n \times m} \times \mathbb{R}^{p \times n}$ where the state, input, and output spaces are $\mathbb{R}^{n}, \mathbb{R}^{m}$, and $\mathbb{R}^{p}$, respectively. Thus the system is governed by the equations

$$
\dot{\boldsymbol{x}}(t)=\boldsymbol{A} \boldsymbol{x}(t)+\boldsymbol{B} \boldsymbol{u}(t),
$$$$
\boldsymbol{y}(t)=\boldsymbol{C} \boldsymbol{x}(t) \text {. }
$$

*Associate Professor, Department of Mathematics and Statistics, Queen's University, Kingston, ON K7L 3N6, Canada

Email: andrewđmast.queensu.ca, URL: http://penelope.mast.queensu.ca/ andrew/
For simplicity, we suppose that $\boldsymbol{B}$ has full rank. Let us first discuss the matter of stabilising this system using sliding mode control, following the conference paper of Hirschorn and Lewis [2003]. In linear sliding mode control, one first defines a sliding surface, in this case a subspace $S$ of the state space $\mathbb{R}^{n}$. This subspace should be chosen to have the following two properties:

1. $S$ is a complement to image $(\boldsymbol{B})$ in $\mathbb{R}^{n}$, i.e., $\mathbb{R}^{n}=\operatorname{image}(B) \oplus S$;

2. the linear map $\boldsymbol{A}_{S}$ on $S$ defined by $\boldsymbol{A}_{S}=\operatorname{pr}_{S} \circ \boldsymbol{A} \circ i_{S}$ is Hurwitz, where $i_{S}: S \rightarrow \mathbb{R}^{n}$ is the inclusion and $\operatorname{pr}_{S}: \mathbb{R}^{n} \rightarrow S$ is the projection along image $(B)$ in the decomposition in part 1 .

In Hirschorn and Lewis [2003] we show that controllability (or, more generally, stabilisability) of $(\boldsymbol{A}, \boldsymbol{B})$ suffices to establish the existence of such a subspace $S$, and we also give a simple algorithm for determining $S$. Note that property 2 above encodes the usual feature that the sliding dynamics be stable. Once one has the sliding surface $S$ at hand, one then designs a variable structure controller that steers trajectories towards $S$ in finite time. This is possible since a sliding surface has property 1 .

Note that the output does not need to participate in stabilisation using sliding mode control. The sliding surface design method of Yan, Spurgeon, and Edwards does involve the output, however. At this stage it is required to know whether a certain MIMO system is stabilisable using static output feedback; this is a problem that is NP-hard. This outputbased design of the sliding surface also requires that there be at least as many outputs as inputs, and a compatibility condition between $\boldsymbol{B}$ and $\boldsymbol{C}$. In the paper, the specific algorithm, involving the output, used to design the sliding surface allows the authors to put the system into a certain form after a linear change of coordinates that is advantageous to their computations. I am not sure whether these advantages can be obtained, after some modification, using a sliding surface not designed using outputs. This might be an interesting matter to investigate. Note, however, that the sliding surface in the paper does have both of the properties above.

The description of linear sliding mode control above suggests a comparison with purely linear design via pole placement, using a scheme as follows:

1. choose closed-loop eigenvalues $\lambda_{1}, \ldots, \lambda_{n-m}$ with generalised eigenspaces $W_{\lambda_{1}}, \ldots, W_{\lambda_{n-m}}$ such that $S \triangleq W_{\lambda_{1}} \oplus \cdots \oplus W_{\lambda_{n-m}}$ is a complement to image $(\boldsymbol{B})$;

2. choose the remaining closed-loop eigenvalues far to the left of the imaginary axis.

The idea is that $S$ plays the rôle of the sliding surface, with the eigenvalues $\lambda_{1}, \ldots, \lambda_{n-m}$ giving the sliding dynamics. The fact that the complementary eigenvalues have large negative real part means that the sliding dynamics will become dominant after a short time. Note that, for linear sliding mode control, the sliding dynamics are achieved after a finite time, whereas, for the pole placement scheme above, the sliding dynamics become dominant after a short time, but are only achieved asymptotically. The distinction becomes even less apparent if one chooses to smooth out the sliding mode control law with some sort of saturation function. Typically the effect of this smoothing is to eliminate chatter at the cost of not achieving the sliding dynamics in finite time. 
We comment that this idea of relating sliding mode control to pole placement is considered using singular perturbation analysis in an early paper of Young, Kokotović, and Utkin [1977].

\section{Linear sliding mode control for nonlinear systems}

A linear sliding mode controller will, just like a purely linear controller, locally stabilise a nonlinear system if it stabilises the system's linearisation. This is well-known, and a geometric proof appears in my conference paper with Hirschorn. Intuitively, one might expect that the more agrressive control action of the sliding mode control law would be more effective at handling the nonlinearities. This does not appear to be unequivocally true. To investigate this, I considered the example used in the paper. I designed a controller/observer as follows.

1. I designed a purely linear controller, using the pole placement algorithm described in the preceding section. Thus the closed-loop system has two eigenvalues equal to the eigenvalues determined by the sliding dynamics of Yan, Spurgeon, and Edwards.

Moreover, the eigenspace for these eigenvalues is exactly the Yan, Spurgeon, and Edwards sliding surface. The remaining two closed-loop eigenvalues were chosen to be -20 . No attempt was made to compensate for uncertainties or nonlinearities in the controller design.

2. I used a standard Luenberger observer, using the observer gain from the paper of Yan Spurgeon, and Edwards. I did not compensate for nonlinearities or uncertainties, although this could easily be done, just as is done in the paper.

In Figure 1 I show a simulation for this setup with the same initial conditions as in the paper I also added some unmatched uncertainty that satisfies the conditions in the paper. I dic not add matched uncertainty, although this could be accomplished with modification to the not compare at all badly with that of Yan, Spurgeon, and Edwards, particularly considering that it is the simplest sort of linear controller.

\section{Anecdotal evidence for the effectiveness of sliding mode control}

Sliding mode control is often attributed with having good robustness properties. I believe this may well be true, although I do not know of any theorems that support a claim like "sliding mode control is more robust than control scheme X." (However, I am also not completely familiar with the literature within which such a claim may be supported.) Nonetheless, my (limited) experiences in our control lab at Queen's suggest that there may be something to the robustness claims of sliding mode control, at least in some cases. In our lab we have a (single and double) pendulum/cart system for which the track on which the cart slides has a significant amount of sticking friction. When performing, say, quadratic regulator design, this sticking friction is not accounted for, and the resulting linear controller, while achieving stabilisation, is quite fragile. Indeed, it is extremely fragile in the double pendulum configuration. Moreover, it takes a significant amount of tuning to get the linear controller to work. By contrast, a linear sliding mode controller implemented on
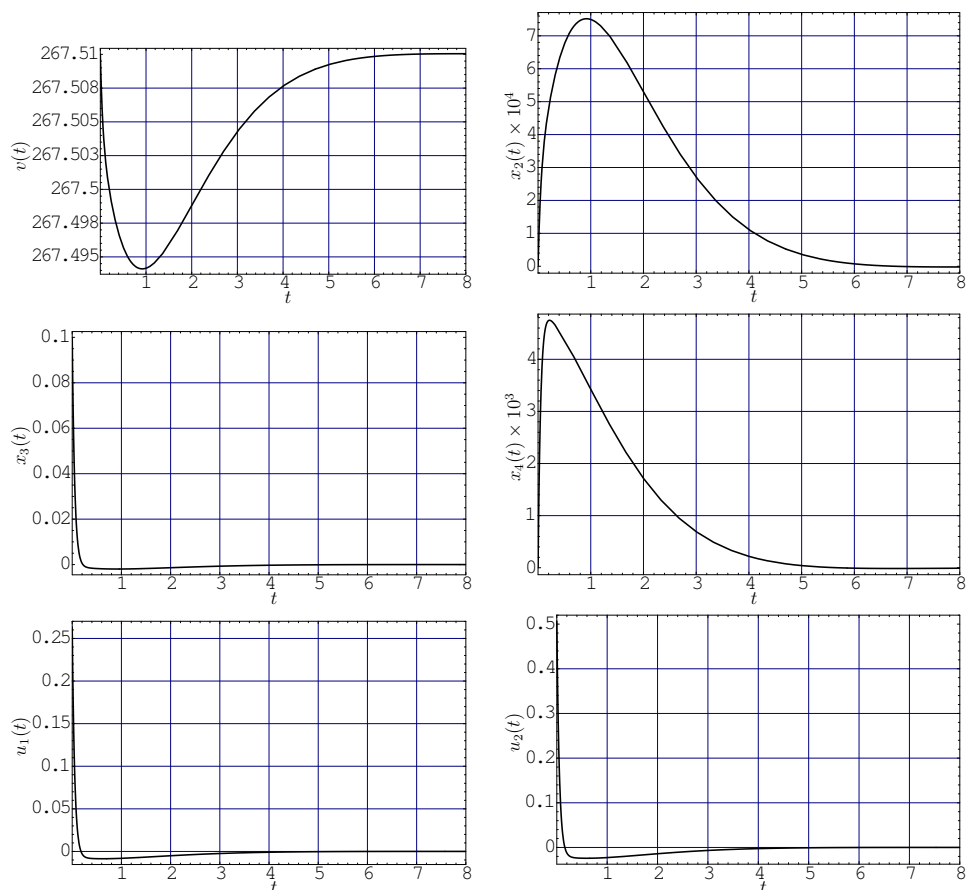

Figure 1. Simulation results for example of Yan, Spurgeon, and Edwards with linear controller and observer: $v(t)=(1+$ $\left.x_{1}(t)\right) v_{0}$ with $v_{0}=267.51$

the same system works with almost no fiddling of the system parameters (e.g., location of sliding mode poles), and is also substantially less fragile. In our lab we also have a torsional vibration apparatus whose behaviour is very linear, i.e., the measured system dynamics closely match the linear model. For this system, it is less easy to see, in a fair comparison, an improvement in performance of sliding mode control design over linear control design.

From this (admittedly extremely limited and not thorough) experience with hardware, I would proffer some rule like, "Sliding mode control is well suited to situations where the aggressive control action is helpful in overcoming the particular character of the unmodelled dynamics." The sticking friction of the inverted pendulum apparatus in our lab is a good example of this. 


\section{Conclusions}

In my discussion, I contend that the matter of comparing sliding mode control with a certain sort of pole placement algorithm is one that is worthy of investigation. At the very least, I think that understanding the relationship between sliding mode control and pole placement is helpful in that it places linear sliding mode control design in a context that is easily understandable. I do believe that sliding mode control can be advantageous in certain sorts of applications. I think that a precise quantification of the sort of application would be interesting. It also seems reasonable to me that, if one adds a standard smoothing function to the sliding mode controller off the sliding surface, then the things that one can do with a linear sliding mode controller are essentially the same as those that can be done with a linear controller, using the scheme of Section 2.

\section{References}

Edwards, C. and Spurgeon, S. K. [1998] Sliding Mode Control: Theory and Applications, volume 7 of Systems and Control Book Series, Taylor \& Francis, London, New York, Philadelphia, Singapore, ISBN 0-748-4060-18.

Hirschorn, R. M. and Lewis, A. D. [2003] Geometric sliding mode control: the linear and linearised theory, in Proceedings of the 42nd IEEE Conference on Decision and Control, pages 2971-2976, Maui, HI

Utkin, V. I. [1992] Sliding Modes in Control and Optimization, Communications and Control Engineering Series, Springer-Verlag, New York-Heidelberg-Berlin, ISBN 0-387-53516-0.

Young, K.-K. D., Kokotović, P. V., and Utkin, V. I. [1977] A singular perturbation analysis of high-gain feedback systems, Institute of Electrical and Electronics Engineers. Transactions on Automatic Control, AC-22(6), 931-939. 